\title{
A Dynamic Data Driven Computational Infrastructure for Reliable Computer Simulations
}

\author{
J.T. Oden ${ }^{1}$, J.C. Browne ${ }^{1}$, I. Babuška ${ }^{1}$, C. Bajaj ${ }^{1}$, L.F. Demkowicz ${ }^{1}$, \\ L. Gray ${ }^{2}$, J. Bass ${ }^{1}$, Y. Feng ${ }^{1}$, S. Prudhomme ${ }^{1}$, F. Nobile ${ }^{1}$, and R. Tempone ${ }^{1}$ \\ 1 Institute for Computational Engineering and Sciences, \\ The University of Texas at Austin, U.S.A. \\ 2 Department of Otolaryngology - Head and Neck Surgery, University of Texas \\ Medical School, University of Texas Health Science Center at Houston, U.S.A.
}

\begin{abstract}
The paper presents an initial study on designing a Dynamic Data Driven (DDD) computational environment to enable reliable and efficient determination of Head Related Transfer Functions (HRTF) for patients with reconstructed ears. The determination involves a synthesis of coordinated experiments and large scale, parallel computer simulations. Time limits (one working day), cost of MRI scans, and parallelism in experimental and computer simulations impose the need for a DDD enabling fast and reliable results.
\end{abstract}

\section{Introduction: Dynamic Data Driven Application Systems}

Traditional application simulations are conducted with static data inputs. In context of Finite Element (FE) simulations, this means defining geometry of the domain of computations, physical coefficients and parameters and source and boundary data. Once the data are specified, the FE codes are run, possibly in an adaptive loop, to assure discretization error control. Any change in data implies restarting the codes from the beginning. In a Dynamic Data Driven Application System [14, an initial set of data is deposited in a depository, but, during the simulation process that follows, the data may evolve or be updated as a result of new measurements, or a feedback mechanism resulting from comparing simulations with experiments. A classical example is a weather prediction system where current simulation results may be updated as new measurements become available "online". Incoming extra information may be incomplete for simply restarting the simulations and more sophisticated "matching mechanisms" are necessary.

Verification and Validation ( $V$ and $V$ ). The systematic study of reliability of computer simulations of physical phenomena is generally divided into two basic processes, Verification and Validation (V and V). As a scientific discipline, V and $\mathrm{V}$ is in its very early stages of development. Verification is the process of determining the accuracy with which a given mathematical model of physics is 
solved; validation is the process of determining that the mathematical model represents the actual physical system with sufficient accuracy. Here verification does not include software engineering issues of the logical correctness of the program which implements the simulations (this is so-called code verification). Verification is thus concerned with estimating and controlling numerical approximation error. In recent years, significant progress has been made in this area. The idea of developing an adaptive control system for implementing $\mathrm{V}$ and $\mathrm{V}$ was made possible by the discovery of the concept of hierarchical modeling and "goal-oriented" adaptive methods based on a posteriori estimates of modeling error [7] and approximation error [1 2]. These error estimates techniques are crucial to the success of any adaptive control paradigm that must react to dynamic data management, and to adapting the models used in simulations.

Uncertainties in Data. Another source of error relevant to validation is due to the randomness of the material and geometry data. There are several approaches available to quantify this error. One direct approach is provided by the perturbation method described in [6]. If it is possible to develop statistical information on the material data to determine the probability density function (PDF), the material coefficients can be represented as a sum of a deterministic average and a stochastic perturbation. A similar decomposition holds then for the solution to the problem. Bounds on the stochastic perturbation of the solution can then be calculated and used as an additional measure of the modeling. The second approach is based on the theory of stochastic functions characterized by Karhunen-Loève expansion (see [4]). Similar methodologies can be used for estimating uncertainties in geometry data, although the problem is tackled in the literature much less frequently.

This paper presents an initial study on designing a Dynamic Data Driven (DDD) computational environment to enable reliable and efficient determination of Head Related Transfer Function 1 (HRTF) for patients with reconstructed ears. The proposed project brings together specialists from Otolaryngology Research Laboratory at the University of Texas Houston Health Science Center, and researchers at the Institute for Computational Engineering and Sciences, at the University of Texas at Austin.

\section{Analysis of the Effect of the Exterior Geometry of the Human Head on Human Hearing}

Historically, many scientific investigations of important physical systems have been purely experimental, out of necessity since the complexities of the systems have been beyond the capability of numerical simulation. An illustrative example of such a system is the human auditory system. A purely empirical approach has placed limitations upon determining basic mechanisms of the sense of hearing, as

\footnotetext{
${ }^{1}$ Pressure on the ear drum expressed as a function of frequency and direction of an incoming plane wave
} 
well as the development of hearing-aid technology for the hearing-impaired, and hearing protection for those working in noisy environments. Application of experimental tools to characterize human hearing have tended to be idiosyncratic to the subject, expensive and time-consuming in application, and dependent upon subjective feedback of the subject being tested. The latter issue is particularly problematic in extremely young patients who lack the ability to fully or productively participate in many testing procedures [513].

We describe how a new approach based on DDDAS in which the numerical modeling capabilities of geometry reconstruction and visualization schemes, and parallel $h p$ boundary element method, developed at ICES, are linked to experimental data obtained at the UT Health Science Center in Houston. The project includes means for controlling modeling and discretization errors. While the technique has a myriad of potential application areas, initial studies are planned to apply the technique to the investigation of the effect of external ear reconstruction in patients born with only one external ear. The sounds that stimulate the ear, and the ability of a subject to localize sound, is dependent in large part on the filtering and amplification characteristics of the external ear, as well as the brain's ability to interpret the person's own HRTF. The work enables the study of sensitivity on the variation of geometry from one external ear, to a surgically-constructed ear, to a normally formed ear and the effects of the variation upon the resultant HRTF. Potential for application of the coupled modeling and experimental technique to other hearing-related issues, and the pursuit of sponsoring of future research are also under investigation.

\section{Geometry Reconstruction from MRI Scans}

In many applications, including the current one, the geometry is too complicated to be modeled by standard CAD systems, and only a linear scan obtained by MRI or CT techniques is available. In the CAD supported case, generation of higher order meshes is possible simply by interpolating the surface parametrization provided by the CAD modeler. In the case of scans, the generation of higher order meshes is not straightforward, since, a priori, only a linear triangulation is available. A number of different procedures are available that will produce higher order meshes from linear triangulations, including element clustering, decimation schemes, etc. A $C^{1}$ surface reconstruction has many advantages from the viewpoint of scattering problems in acoustics, including the elimination element clustering around artificial $C^{0}$ edges, and a lowering of the order of singularities in the integral kernels. One approach for generating a $C^{1}$ representation from a linear triangulation has been given by Bajaj, Xu, 3].

The $C^{1}$ geometry reconstruction, done within a Geometrical Modeling Package, a small CAD system developed at ICES that interfaces with the boundary element codes, is discussed next, and allows for an initial mesh generation as well automatic geometry updates during mesh refinements. 


\section{Modeling of Human Head Acoustics}

The acoustics of the human head is modeled with a comparatively simple linear acoustics model which, in the frequency domain, reduces to solving the Helmholtz equation in the domain exterior to the human head (but including the ear channel), accompanied by Sommerfeld radiation condition at infinity, and impedance boundary conditions. The material data are represented by impedance, usually assumed to be piecewise constant, with different values for the head, ear channel, and the ear drum. The values may depend (mildly) on the patient, i.e. the impedance of the head may reflect the amount of patient's hair.

\section{Parallel $h p$-Adaptive Boundary Elements}

Major components of the parallel $h p$ BEM code developed to solve the acoustics problem, are given below.

Burton-Miller Integral Equation (BMIE). The Helmholtz equation is replaced with an equivalent Burton-Miller integral equation defined on the surface of the head, including the ear channel and the ear drum. The formulation is uniformly stable in frequency. The integral equation is formulated in a variational form, allowing for reduction of singularities to weak singularities only. This facilitates and accelerates the integration of element matrices.

$h p$-discretizations. The integral equation, in its variational form, is discretized then using Galerkin approximation and $h p$ finite element meshes in which element size $h$ and polynomial order $p$ may be varied locally to minimize the error per given problem size. If done properly, the $h p$ discretizations have the potential of delivering exponential convergence and superaccurate results.

Goal-oriented adaptivity. The adaptivity is driven by minimizing the error in a specific quantity of interest, in our case - the average pressure on the ear drum. The algorithm requires solving simultaneously both the original and dual problems [11. The error in pressure is then represented in terms of a functional involving both solutions, and serves as a starting point to determine optimal $h p$ mesh refinements. The algorithm produces a sequence of meshes of increasing size and complexity with corresponding solutions, i.e. the pressure distribution on the head. The problem involves multiple loads as one mesh serves all (typically sixty) directions of the incoming plane wave. The problem can thus be very time consuming, and even with a parallel implementation, attaining a 1 percent accuracy, may require at this point many CPU hours. During the mesh optimization process, the code deposits intermediate meshes and corresponding (multiple) solutions on the disk from which the results may be accessed and visualized. 
Parallel implementation. The parallel implementation has been done within PLAPACK [10]. This includes integration of element matrices, a dense linear solver based on LU decomposition, and a posteriori error estimation done in parallel. The problem does not involve any domain decomposition; a copy of data structure supporting the geometry, mesh and solution, is maintained on each processor.

Error control. At present, the code includes only an a-posteriori error control of the discretization error. Neither modeling nor geometry induces errors are being estimated. Sensitivity of the solution with respect to the geometry is critical in the case of frequencies corresponding to resonating modes (concha or ear channel modes).

\section{A DDDAS for Modeling Acoustics of Human Head}

A DDD adaptive control system is under study in which scanned images of external ears (both premature and reconstructed) is to be provided from remote repository at the UT Health Sciences Center at Houston. The team in Austin constructs mathematical models of sound propagation into the ear canals of specific patients. The computed HRTF's are then to be reported back to Houston to allow for a comparison with those obtained experimentally.

Existing computational methodology are being upgraded to a fully dynamic system enabling a continuous exchange of data between Houston and Austin, and a critical comparison of the computational and experimental results. A typical patient visit to the Houston facility lasts one day (eight working hours), and the targeted mode of operation is to complete the patient analysis within that time.

A schematic mode of operation is presented in Fig. 1. All software including visualization will be run in a client/server mode, with the actual codes being executed at ICES platforms. A common depository will be established with a new directory opened for each new patient. At any time during the operation, the depository may include the following files.

MRIscan1. The first MRI scan of the patient head and ear channel.

MRIscan2. The second MRI scan, done later, if the geometry reconstruction code fails to deliver acceptable results.

head_geometry. Patient head reconstructed geometry, based on one or two MRI scans.

Results of computation. A sequence of optimal meshes with corresponding pressure distributions and computed HRTFs.

Error estimates. Results of sensitivity analysis providing error bounds for obtained numerical results (for each mesh and each frequency), as well as possible results of comparison of measured and computed HRTFs.

The following is a possible scenario. A patient arrives at the Houston facility early in the morning, the first MRI scan $(\$ 1,000$ each) is made and deposited in 


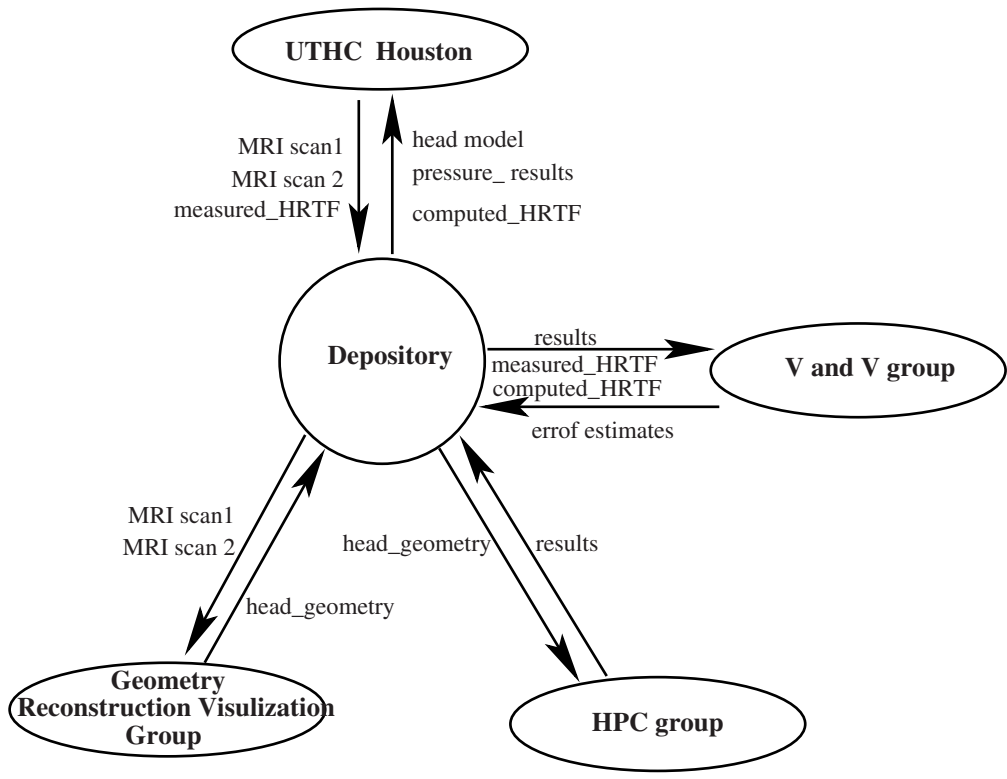

Fig. 1. A schematic idea of the proposed DDD computational environment

the depository. The initiation of a new patient case includes also specifying the corresponding impedance data.

The geometry of the patient is reconstructed in the form of a fine, unstructured linear mesh and stored in the depository. The reality of the geometry reconstruction schemes is that, in presence of noisy data, they produce many artifacts. Such discrepancies prove to be critical in highly accurate computer simulation of acoustics resulting in erroneous results. The model has to be "cleaned" interactively. The corrections will be done in communication between Houston and the geometry reconstruction group at Austin. The process may result in the necessity of taking another MRI scan and producing a better resolution geometry representation based on both sets of data.

The initial, "dirty" image is still sufficient to start the solution process and begin the mesh optimization process. As new, cleaner data arrive in the depository, the geometry input will be upgraded in the ongoing numerical simulations. One should emphasize that several 2 copies of the code will be executed in parallel.

The moment first meshes are generated and results are computed and stored in the depository, the Validation and Verification group begins analyzing sensitivity of the results with respect to geometry and impedance data, as well as more thorough (and expensive) discretization error estimates are computed (initial, rough estimates are provided by mesh optimization algorithms).

In meantime, the team in Houston completes determining HRTFs experimentally and the results are communicated to the V\&V group for a final, quantitative

\footnotetext{
${ }^{2}$ The HRTFs are usually determined for 6-10 different frequencies
} 


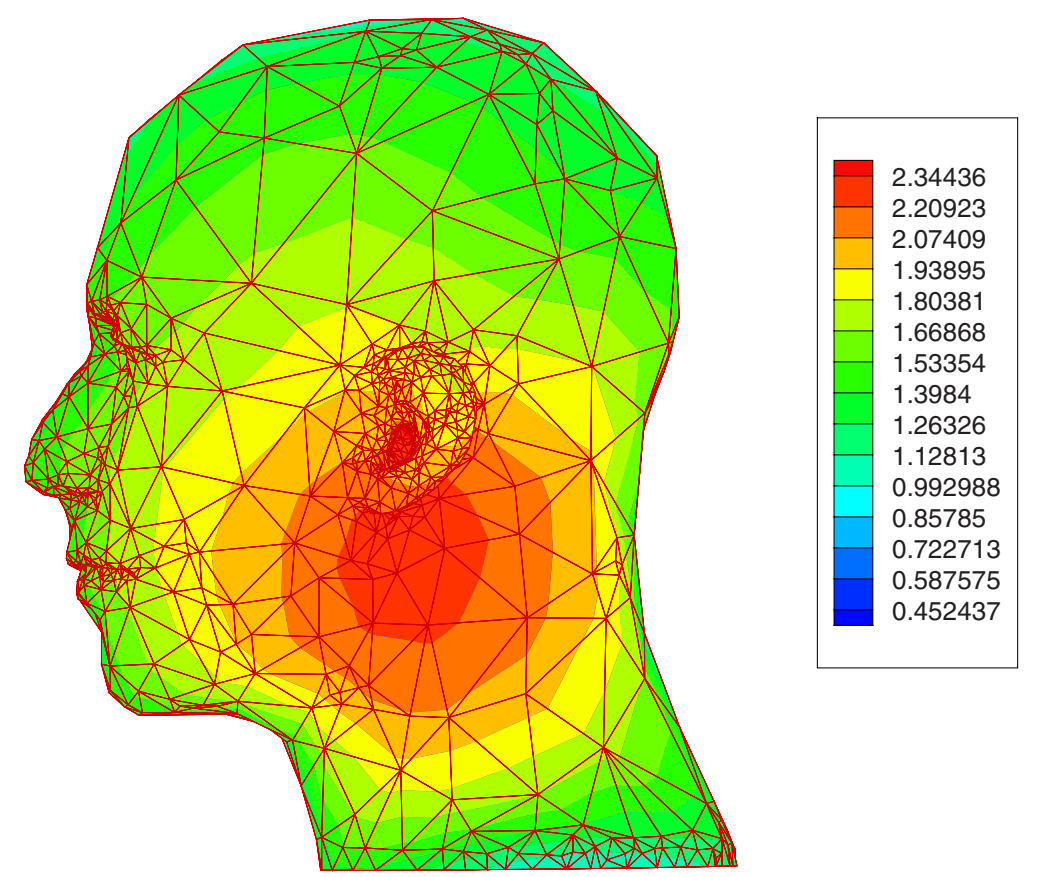

Fig. 2. Pressure distribution on a model of human head

error analysis. Dependently upon results of the sensitivity analysis, the group at Houston may decide to change the impedance data, as well.

A new methodology for comparing the experiments with simulations is needed. This will involve comparing not only the measured and computed HRTF's but also designing experiments that may verify the computed data in an "inverse mode". For example, we can test the accuracy of vertical and horizontal sound localization using established "auditory virtual reality" techniques. To do this we use the calculated HRTF's from reconstructed ears to make special stereo sounds and determine if localization under headphones is accurate.

\section{Conclusions}

The project described is an excellent example of an important DDDAS. The system builds on collaborations of medical researchers, computer scientists, computational scientists, engineers, and mathematicians in Houston and Austin. On top of necessary improvements of existing methodologies, the main challenges are:

- to build an environment allowing for a dynamic data transfer and simultaneous visualization of results by the three involved parties (experiment, geometry reconstruction and visualization, modeling), and, 
- to develop algorithms for estimating sensitivity of the simulated results with respect to reconstructed geometry and material data,

- to establish a mathematically sound methodology that would allow for comparing the experiments and simulations with a feedback and a dynamic modification of data on both sides.

Acknowledgment. The authors gratefully acknowledge support of the NSF under grant 0205181 .

\section{References}

1. Ainsworth, M., and Oden, J.T.: A Posteriori Error Estimation in Finite Element Analysis. John Wiley \& Sons, New York, (2000)

2. Babuška, I., Strouboulis, T.: Finite Element Method and its Reliability. Oxford Univ. Press (2001)

3. Bajaj, C., Chen, J., and Xu, G.: Modeling with cubic A-patches, ACM Transactions on Graphics, 14 (2) (1995) 103-133.

4. Deb, D.K., Babuška, I., Oden, J.T.: Solution of Stochastic Partial Differential Equations Using Galerkin Finite Element Techniques. Comput. Methods Appl. Mech. Eng. 190 (2001), 6359-6372

5. Jahrsdoerfer, R.A., Yeakley, J.W., Aguilar, E.A., Cole, R.A., and Gray, L.C.: A grading system for the selection of patients with congenital aural atresia. American Journal of Otolology 13 (1992) 6-12

6. Kleiber, M., Hien, T.D.: The Stochastic Finite Element Method: Basic Perturbation Technique and Computer Implementation. John Wiley \& Sons, (1992)

7. Oden, J.T., Prudhomme, S., Estimation of modeling error in computational mechanics. J. Comput. Phys., 182 (2002), 496-515

8. Oden, J.T., Babuska, I., Nobile, F., Feng, Y., and Tempone, R.: Theory and methodology for estimation and control of errors due to modeling, approximiation, and uncertainty. Computer Methods in Applied Mechanics and Engineering, MAFELAP 2003 Special issue (to appear)

9. Oden, J.T., Browne, J.C., Babuska, I., Liechti K.M., Demkowicz, L., Bass J., Feng, Y., Prudhomme, S., Nobile, F., Tempone, R.: A computational infrastructure for reliable computer simulations. In F. Darema (ed.) Dynamic Data Driven Application Systems, Kluver Academic Publishers (to appear)

10. van de Geijn, R.A.: Using PLAPACK, The MIT Press (1997)

11. Walsh, T., Demkowicz, ,L.: $h p$ Boundary element modeling of the external human auditory system - goal oriented adaptivity with multiple load vectors, Computer Methods in Applied Mechanics and Engineering, 192 (2003) 125-146

12. Walsh, T., Demkowicz, L, Charles, R.: Boundary element modeling of the external human auditory system, JASA (to appear)

13. Wilmington, D., and Gray, L., Jahrsdoerfer, R.: Binaural processing after corrected congenital unilateral conductive hearing loss. Hearing Research, 74 (1994) 99-114

14. NSF Workshop (2000) http://www.cise.nsf.gov/dddas 